\title{
TBILISI RADIOCARBON DATES IV
}

\author{
A A BURCHULADZE and G I TOGONIDZE
}

Radiocarbon Laboratory, Tbilisi State University, Tbilisi, USSR, 380028

Results presented below were obtained by the Radiocarbon Laboratory of Tbilisi State University from 1976 to 1983. Throughout that period dates were determined not only for archaeologic samples but also for samples of mineral waters, soil humus, and geologic origin. Georgian wines of 1909-1975 were also analyzed but the results obtained are not discussed in this paper as they were published elsewhere (Burchuladze et al, 1977, 1979, 1980, 1982).

Dates of the studied samples were obtained by measuring the ${ }^{14} \mathrm{C}$ activity on a 3-channel SL-30 Intertechnique Liquid Scintillation Spectrometer. Working parameters of each channel were determined by manual adjustment of the discrimination threshold. In our procedure the maximally determined age was equal to 45,500 years. The age of individual samples was determined as the mean value of data obtained in three individual channels.

Scintillation solvent was synthesized in the all-purpose physico-chemical unit designed at Tbilisi State University which provides high reaction yields of $\mathrm{CO}_{2}-100 \%, \mathrm{CH}_{2}-98 \%$, and $\mathrm{C}_{6} \mathrm{H}_{6}-97 \%$ for stoichiometric values (Burchuladze et al, 1970, 1974, 1979). The background in individual channels of SL-30 was determined, using CHEMAPOL spectroscopic benzene (Czechoslovakia) for solution volume of $10 \mathrm{ml}$; $5 \mathrm{ml}$ of scintillation solution synthesized from the sample were diluted to $10 \mathrm{ml}$ by background benzene of the same grade.

As modern ${ }^{14} \mathrm{C}$ standard we used intermediate standard prepared in the Radiocarbon Laboratory of Geology Institute, Academy of Sciences, USSR, the activity of which was corrected by the NBS oxalic acid standard (USA).

In our calculations the value $T=5730 \pm 40$ years was assumed for the half-life of ${ }^{14} \mathrm{C}$. It should also be noted that in some instances final data on the ${ }^{14} \mathrm{C}$ activity of the sample were corrected according to ${ }^{13} \mathrm{C}$.

We present results on the absolute age of archaeologic samples obtained by the radiocarbon method as well as data with corrections made by the conversion table (Damon et al, 1972).

\section{ARCHAEOLOGIC SAMPLES}

\section{Arakhlo series, Georgian SSR}

Institute of History, Archaeology and Ethnography, Acad Sci GSSR, in collaboration with Fine Arts Mus is investigating Arakhlo I, an early Neolithic agric mound. It is a Gora-Tepe type settlement near Arakhlo village, Bolnisi dist, East Georgia. Test trenches were excavated both in the inhabited hill (6m high) and vicinity. In addition to the main settlement, excavations revealed 2 ditches of different periods encircling the hill on 3 sides, a settlement represented by shelters half dug out in the foot of the hill, and synchronous burials. The settlement presently shows 7 building horizons; 
the upper 6 consist of structures made of air-dried bricks; the lowest horizon only shows narrow trenches dug into ground.

Archaeol dating of the main layers is 6 th to 5 th millennium BC. Samples subm by T Chubinishvili (TB-89, -92, -277) and D Gogelia (TB-300, -309, $-331)$.

TB-89. Arakhlo I

$2030 \pm 40$

Charcoal, depth $0.7 \mathrm{~m}$, annex structure no. 4 , $1 \mathrm{st}$ bldg horizon, from early agric settlement of inhabited hill; site is presently on state farm on Arakhlo village. Est cal age $=2000 \pm 49 \mathrm{BP}$.

\section{TB-92. Arakhlo I}

$6720 \pm 60$

Charcoal, depth $0.9 \mathrm{~m}$, under 1 st floor of structure no. 19, $1 \mathrm{st}$ bldg horizon. Est cal age $=7340 \pm 73 \mathrm{BP}$.

\section{TB-277. Arakhlo I}

$6970 \pm 60$

Charcoal, depth $1.6 \mathrm{~m}, \operatorname{Tr} 1, \mathrm{~N}$ sec.

TB-300. Arakhlo I

$7350 \pm 70$

Charcoal, depth 5.6m, Tr 2, 7th bldg horizon.

TB-309. Arakhlo I

$6980 \pm 70$

Charcoal, depth $4.85 \mathrm{~m}, \operatorname{Tr} 16$, structure no. 44 .

TB-331. Arakhlo I

$6600 \pm 140$

Charred organic matter from depth 1.85, 2nd bldg horizon. Est cal age $=7190 \pm 73 \mathrm{BP}$.

\section{Gebi series}

Samples are from ancient copper and antimony mines and metallurgic slag heaps near Gebi village in Highland Racha, Oni dist, Georgian SSR, except Tvibrasheri sample (TB-304) from ancient copper mine of Highland Abkhazia, upper Kodori R, S slope of Caucasian range. This mine has geol, technol and hist features similar to Gebi mines.

Samples subm by T Mujiri, K Kalandadze, G Gobejishvili, G Imnashvili, B Maisuradze, I G Korinteli, Mining Mechan Eng Inst, Acad Sci GSS, and Archaeol Inv Center, Acad Sci GSSR.

\section{TB-90. Gona}

$2880 \pm 40$

Wood, depth $1 \mathrm{~m}$, part of wooden lining (prop) from copper mine, Chkornaliani deposits of settlement Gona, Gebi village, in horizontal underground mine $40 \mathrm{~m}$ from mine entry; mine was filled with slag and running water. Est cal age $=3010 \pm 80 \mathrm{BP}$.

\section{TB-91. Gona}

$2910 \pm 45$

Wood, depth $1 \mathrm{~m}$, part of wooden trough; loc, mine, and conditions similar to TB-90. Est cal age $=3050 \pm 80 \mathrm{BP}$. 
TB-255. Zopkhito

Charcoal from underground antimony mine, coll under caved-in rock, Zopkhito deposit, Gebi village. Est cal age $=3100 \pm 80 \mathrm{BP}$.

TB-302. Zopkhito

$3200 \pm 170$

Charcoal from underground mine depth $20 \mathrm{~m}$ from surface, vein no. 6 , adit no. 80 , antimony mine. Est cal age $=3400 \pm 125 \mathrm{BP}$.

TB-304. Tvibasheri, Abkhazian ASSR

$3100 \pm 70$

Wood, depth $20 \mathrm{~m}$, part of timbers from copper mine, Tvibasheri deposit, upper Kodori R. Est cal age $=3300 \pm 125 \mathrm{BP}$.

TB-310. Sagebi

$3630 \pm 50$

Charcoal from metallurgic slag, Sagebi village, left bank of Zopkhitura R. Est cal age $=3950 \pm 63 \mathrm{BP}$.

TB-334. Sagebi

$3590 \pm 60$

Charcoal, depth $1 \mathrm{~m}$, from antimony mine, left bank of Zopkhitura R. Est cal age $=3890 \pm 63 \mathrm{BP}$.

TB-333. Uravi

$1470 \pm 50$

Charcoal from metallurgic slag, depth $0.7 \mathrm{~m}$, right bank of Lukhuni Water R, Uravi village, Ambrolauri dist, Upper Racha. Est cal age $=1400 \pm$ 22BP.

TB-335. Khirkhi

$3120 \pm 50$

Charcoal from antimony mine, Khirkhi deposit, Gebi village, right bank of Zopkhitura R. Est cal age $=3300 \pm 125 \mathrm{BP}$.

\section{Great Mtskheta series, Georgian SSR}

At end of 4th century BC Kartlian (Iberian) Kingdom with Mtskheta as its capital formed in E Georgia. Great Mtskheta, proof of which is documented in ancient writings, implies city and its vicinity. This series includes dates of wood and charcoal samples from Mtskheta dist. Hill-like settlement Samtavro I is situated within boundaries of contemporary town and dates from 12th to 6 th centuries BC. Kalandadzisgora settlement of Narekvavi village has approximately same dates.

Samadlo is urbanized settlement belonging to early Hellenistic Age (4th to 3rd centuries BC) on right bank of Mtkvari R, W of Dzegvi village.

Fence with towers and counterforts built of air-dried bricks were recently found $\mathrm{N}$ of contemporary town on right bank of Aragvi $\mathrm{R}$ and is important for studying hist topography of ancient Mtskheta. Early feudal mounds were uncovered on farmland near Tsilkani village $\mathrm{N}$ of Mtskheta.

Samples subm by A Apakidze (head), A Kalandadze, Yu Gagoshidze, V Nikolaishvili, and T Giunashvili, Archaeol Inv Center, Acad Sci GSSR. 
TB-244. Samtavro I 125BP.

Charcoal, depth $1 \mathrm{~m}$, remains of structure no. 1 . Est cal age $=3200 \pm$

TB-245. Samtavro I

$2950 \pm 50$ 80BP.

Charcoal, depth $2 \mathrm{~m}$, remains of structure no. 2. Est cal age $=3100 \pm$

TB-258. Samtavro I

$\mathbf{3 0 3 0} \pm \mathbf{5 0}$

Charcoal, depth $1.5 \mathrm{~m}$, remains of floor of burned structure. Est cal age $=3200 \pm 125 \mathrm{BP}$.

TB-259. Samtavro I

$\mathbf{2 5 6 0} \pm \mathbf{5 0}$

Charcoal, depth 2.5 to $3 \mathrm{~m}$, remains of floor of burned structure no. 5 , 1 st bldg level. Est cal age $=2600 \pm 102 \mathrm{BP}$.

TB-271. Samtavro I

$2570 \pm 40$

Charcoal, depth 2 to 2.5 m, refuse pit no. 3, 3rd bldg level. Est cal age $=2600 \pm 102 \mathrm{BP}$.

TB-290. Samtavro I

$2610 \pm 60$

Charcoal, depth 0.6 to $1 \mathrm{~m}$, fragment of wooden pillar of structure no. 7. Est cal age $=2700 \pm 102 \mathrm{BP}$.

TB-278. Gartiskari

$2430 \pm 40$

Charcoal, depth $1.8 \mathrm{~m}$, from cultural layer of ancient fortification. Est cal age $=2460 \pm 39 \mathrm{BP}$.

TB-327. Gartiskari

$2230 \pm 50$

Charcoal, depth $2 \mathrm{~m}$, remains of wooden beams of interior floor of square tower. Est cal age $=2230 \pm 49 \mathrm{BP}$.

TB-418. Gartiskari

$2590 \pm 50$

Charcoal, depth $2 \mathrm{~m}$, remains of fallen wooden pillar in tower no. 2. Est cal age $=2700 \pm 102 \mathrm{BP}$.

TB-224. Narekvavi

$2430 \pm 40$

Charcoal, depth $1.7 \mathrm{~m}$, remains of structure, Narekvavi village, $5 \mathrm{~km}$ from Mtskheta. Est cal age $=2460 \pm 39 \mathrm{BP}$.

TB-339. Narekvavi

$2450 \pm 50$

Charcoal, depth $1.3 \mathrm{~m}$, remains of structure no. 1, settlement Kalandadze gora-II. Est cal age $=2490 \pm 39 \mathrm{BP}$.

TB-94. Samadlo I

$2300 \pm 40$

Wood, depth $3 \mathrm{~m}$, piece of beam from stone structure with counterforts, 1 st bldg horizon, Dzegvi village. Est cal age $=2310 \pm 39 \mathrm{BP}$. 
TB-95. Samadlo I

$2280 \pm 40$

Wood, depth $1 \mathrm{~m}$, piece of beam from $\mathrm{N}$ stone wall of crypt, $1 \mathrm{st}$ bldg horizon, Dzegvi village. Est cal age $=2280 \pm 39 \mathrm{BP}$.

TB-96. Samadlo III

$2390 \pm 40$

Wood, depth $0.7 \mathrm{~m}$, piece of beam from stone of pre-Hellenistic layer, Dzegvi village. Est cal age $=2420 \pm 39 \mathrm{BP}$.

TB-305. Tsilkani

$\mathbf{1 8 9 0} \pm \mathbf{5 0}$

Charcoal, depth $2.2 \mathrm{~m}$, refuse pit no. 1 , Tsilkani village, 9 to $10 \mathrm{~km}$ from Mtskheta. Est cal age $=1850 \pm 33 \mathrm{BP}$.

\section{Shenako series}

Samples from Highland-Tushetian Archaeol Expedition of Nishtako hill, Shenako village, Highland Tushetia, Akhmeta dist, Georgian SSR. Highland Tushetia is on $\mathrm{N}$ slope of Caucasian range, bordered on $\mathrm{E}$ by Daghestan ASSR and on N by Checheno-Ingush ASSR. Shenako terraced settlement was found on Nishtako hill, typical of mountainous regions, with cultural structures of different periods, including iron-smelting workshop. Lower layers of mound contain hitherto unknown mountain cultures. Samples subm by R Dolaberidze, State Mus Georgia.

TB-221. Shenako

$1680 \pm 50$

Charcoal, depth $0.8 \mathrm{~m}$, Sq SD-43, 2nd layer, 1st terrace, SW slope, Nishtako hill. Est cal age $=1620 \pm 41 \mathrm{BP}$.

TB-222. Shenako

$1640 \pm 50$

Charcoal, depth $0.6 \mathrm{~m}$, Sq SD-42, 2nd layer, 1 st terrace, SW slope. Est cal age $=1580 \pm 41 \mathrm{BP}$.

TB-229. Shenako

$1970 \pm 40$

Charcoal, depth 1.3m, Sq SD-43, 3rd layer, 1st terrace, SW slope. Est cal age $=1940 \pm 33 \mathrm{BP}$.

TB-267. Shenako

$2140 \pm 40$

Charcoal, depth 1.7 to $1.8 \mathrm{~m}$, Sq SD-32, 3rd layer, 1st terrace, SW slope. Est cal age $=2120 \pm 49 \mathrm{BP}$.

TB-268. Shenako

$1990 \pm 45$

Charcoal, depth 2m, Sq SD-33, 3rd layer, 1st terrace, SW slope. Est cal age $=1960 \pm 33 \mathrm{BP}$.

TB-269. Shenako

$2090 \pm 45$

Charcoal, depth 1.4m, Sq SE-83, 3rd layer, 2nd terrace, SW slope. Est cal age $=2070 \pm 49 \mathrm{BP}$. 
TB-270. Shenako

Charcoal, depth $2 \mathrm{~m}, \mathrm{Sq}$ SD-35, 3rd layer, 1 st terrace, SW slope. Est cal age $=2000 \pm 49 \mathrm{BP}$.

TB-311. Shenako

$1710 \pm 40$

Charcoal, depth $1.3 \mathrm{~m}$, remainder of wall, $\mathrm{N}$ slope. Est cal age = $1650 \pm 41 \mathrm{BP}$.

TB-312. Shenako

$760 \pm 40$ 39вP.

Charcoal, depth $0.3 \mathrm{~m}$, remainder of wall, $\mathrm{N}$ slope. Est cal age $=730 \pm$

TB-313. Shenako

$830 \pm 40$

Charcoal, depth $0.3 \mathrm{~m}$, remainder of wall, crest of Nishtako hill. Est cal age $=800 \pm 39 \mathrm{BP}$.

TB-314. Shenako

$1000 \pm 45$

Charcoal, depth $1.5 \mathrm{~m}$, remainder of wall, blacksmith's shop, crest of Nishtako hill. Est cal age $=950 \pm 39 \mathrm{BP}$.

\section{Kobuleti series, Adjarian ASSR}

Eastern Black Sea precoastal region (now W Georgia) was part of ancient Oikumena, where productive society originated very early. Later, rather advanced farming culture developed here and gave rise to famous Colchian civilization.

Group of archaeol mounds of several periods was found $\mathrm{N}$ and $\mathrm{E}$ of Kobuleti resort, on banks of Ochkhamuri and Chorokhi Rivers. Group includes early farming two-layer settlement, Ispani, underlying $2 \mathrm{~m}$ peat, and multi-layer Namcheduri settlement which chronologically and culturally follows Ispani settlement from end of 4th millennium BC to end of 3rd or beginning of 2 nd millennium BC. Samples subm by D A Khakhutaishvili, Archaeol Exped SW Georgia, Batumi Research Inst, Acad Sci GSSR.

TB-231. Ispani

$3380 \pm 45$

Wood, depth $1.3 \mathrm{~m}$ from lower layer of peat covering Ispani settlement, $1 \mathrm{~km}$ from town Kobuleti. Est cal age $=3600 \pm 103 \mathrm{BP}$.

TB-232. Ispani

$4130 \pm 50$

Wood, depth $2.4 \mathrm{~m}$, upper layer of settlement from same loc as TB231. Est cal age $=4600 \pm 108 \mathrm{BP}$.

TB-233. Ispani

$4590 \pm 60$

Wood, depth 2.8 to $3 \mathrm{~m}$, lower layer of settlement from same loc as TB-231. Est cal age $=5130 \pm 92 \mathrm{BP}$.

\section{TB-230. Namcheduri}

$3130 \pm 45$

Coal, depth $4.5 \mathrm{~m}$, Sq 17, 6th layer, Sec III, ancient Colchian settlement, right bank of Ochkhamuri R, Namchedura hill, town Kobuleti. Est cal age $=3300 \pm 125 \mathrm{BP}$. 
TB-306. Namcheduri

Wood, Sec SO, Sq 25, 7th layer, from same loc as TB-230. Est cal age = $3700 \pm 103 \mathrm{BP}$.

\section{TB-307. Namcheduri}

$2960 \pm 60$

Coal, SW sec, Sq 33, 4th layer, from same loc as TB-230. Est cal age = $3110 \pm 80 \mathrm{BP}$.

\section{TB-319. Namcheduri}

$2710 \pm 40$

Wood, depth $2.5 \mathrm{~m}$, SW sec, Sq 39, 3rd layer, from same loc as TB-230. Est cal age $=2800 \pm 102 \mathrm{BP}$.

TB-320. Namcheduri

$2960 \pm 45$

Wood, depth $3.5 \mathrm{~m}, \mathrm{SW}$ sec, Sq 26, 5 th layer, from same loc as TB-230. Est cal age $=3110 \pm 80 \mathrm{BP}$.

TB-321. Namcheduri

Wood, depth $4 \mathrm{~m}$, SW sec, Sq 26, 5th layer, from same loc as TB-230. Est cal age $=3020 \pm 80 \mathrm{BP}$.

TB-323. Namcheduri

Wood, depth 7m, SW sec, Sq 11, 6th layer, from same loc as TB-230. Est cal age $=3600 \pm 103 \mathrm{BP}$.

TB-324. Namcheduri

$3000 \pm 60$

Wood, depth $8 \mathrm{~m}$, SW sec, Sq 2, 6th layer, from same loc as TB-230. Est cal age $=3200 \pm 125 \mathrm{BP}$.

\section{TB-332. Namcheduri}

Charred cereals (corn), depth $5 \mathrm{~m}$, SW sec, Sq 17, 5 th layer, from same loc as TB-230. Est cal age $=3060 \pm 80 \mathrm{BP}$.

\section{Askana, Mziani, and Charnali series}

According to legend, Colchian tribes, in particular, Khalides were among first discoverers of iron and steel manufacture. In recent years, in foothills of E Black Sea coastal region, $i e$, ancient Colchis, archaeologists have found center of ancient iron metallurgy, with production sites yielding ca 400 artifacts. Askana II and Mziani from Askana and Mziani villages, respectively, Makharadze dist, Georgian SSR, are oldest workshops excavated here. Another workshop, Charnali, is in gorge of Charnali R, left tributary of Chorokhi R, Khelvachauri dist, Adjarian ASSR. Samples subm by D A Khakhutaishvili.

TB-234. Askana II

$3180 \pm 45$

Coal, depth 0.5 , from bottom of iron-smelting furnace no. 1, upper Tskaltsitela R. Est cal age $=3400 \pm 125 \mathrm{BP}$.

TB-235. Askana II

$3080 \pm 45$

Coal, depth $0.5 \mathrm{~m}$, from bottom of iron-smelting furnace no. 2 , from same loc as TB-234. Est cal age $=3300 \pm 125 \mathrm{BP}$. 
TB-401. Mziani II

Coal, depth $0.3 \mathrm{~m}$, from iron-smelting furnace, Sample 1. Est cal age $=$ $2600 \pm 102 \mathrm{BP}$.

TB-402. Mziani II

$2890 \pm 50$

Coal, depth $0.5 \mathrm{~m}$, from iron-smelting furnace, Sample 2, from same loc as TB-401. Est cal age $=3020 \pm 80 \mathrm{BP}$.

TB-403. Mziani II

$\mathbf{3 2 3 0} \pm \mathbf{5 0}$

Coal, depth $1.2 \mathrm{~m}$, from floor of iron-smelting furnace, Sample 3, from same loc as TB-401. Est cal age $=3400 \pm 125 \mathrm{BP}$.

TB-404. Mziani III

$\mathbf{2 5 4 0} \pm \mathbf{5 0}$

Coal, depth, $0.8 \mathrm{~m}$ from floor level of iron-smelting furnace, Sample 1, from same loc as TB-401. Est cal age $=2600 \pm 102 \mathrm{BP}$.

TB-405. Mziani III

$\mathbf{2 8 5 0} \pm \mathbf{5 0}$

Coal, depth $0.9 \mathrm{~m}$ from floor level of iron-smelting furnace, Sample 2, from same loc as TB-401. Est cal age $=2970 \pm 80 \mathrm{BP}$.

TB-406. Mziani III

$250 \pm 45$

Coal, depth $0.3 \mathrm{~m}$ from ground surface, charred board of table top, Sample 3, from same loc as TB-401. Est cal age $=290 \pm 42 \mathrm{BP}$.

TB-407. Mziani IV

$2510 \pm 50$

Coal, depth $0.5 \mathrm{~m}$, from iron-smelting furnace, Sample 1, from same loc as TB-401. Est cal age $=2600 \pm 102 \mathrm{BP}$.

TB-408. Mziani IV

$3170 \pm 45$

Coal, depth $0.3 \mathrm{~m}$, from base of pit of iron-smelting furnace, Sample 2, from same loc as TB-401. Est cal age $=3400 \pm 125 \mathrm{BP}$.

TB-286. Charnali II

$2670 \pm 50$

Coal, depth $1.5 \mathrm{~m}$, from bottom of iron-smelting furnace no. 1, Charnali site, $S$ slope of gorge of Charnali R. Est cal age $=2700 \pm 102 \mathrm{BP}$.

TB-287. Charnali II

$2720 \pm 50$

Coal, depth $1.4 \mathrm{~m}$, from bottom of iron-smelting furnace no. 2, Charnali, E Slope, from same loc as TB-286. Est cal age $=2800 \pm 102 \mathrm{BP}$.

TB-288. Charnali III

$2750 \pm 50$

Coal, depth $1.4 \mathrm{~m}$ from bottom of iron-smelting furnace no. 1, Charnali site, E slope, from same loc as TB-286. Est cal age $=2850 \pm 80 \mathrm{BP}$.

\section{Kakheti series}

Kakheti is in E Georgia on lower slopes of Caucasian range. This series includes samples coll at four sites: 1) Khramebi site, Nukriani village, Sig- 
nakhi dist, burial mound of Gombori range; 2) burial Gora I, Alazani valley, Tsnori dist; 3) Pevrebi site, Melani village, Gurjaani dist, pit burial of lower tier of cemetery, S slope of Gombori range; 4) Udabno site, burial mound no. 1, right bank of Iori R, Udabno village; Zeiani site, Manavi village, Sagarejo dist. Samples subm by Sh Dedabrishvili (TB-242, -243) and K Pitskhelauri, Kakhetian Archaeol Expedition, Archaeol Inv Center, Acad Sci GSSR.

TB-242. Khramebi

$$
4150 \pm 50
$$

Wood, depth $3 \mathrm{~m}$, fragment of wooden cover of burial mound. Est cal age $=4600 \pm 108 \mathrm{BP}$.

TB-243. Gora I

$$
4110 \pm 50
$$

Wood, depth $2.5 \mathrm{~m}$, fragment of wooden cover of burial mound. Est cal age $=4500 \pm 108 \mathrm{BP}$.

TB-247. Pevrebi

$3230 \pm 45$

Wood, depth $2 \mathrm{~m}$, fragment of wooden cover of pit burial no. 15. Est cal age $=3400 \pm 125 \mathrm{BP}$.

TB-248. Pevrebi

$3250 \pm 45$

Wood, depth $1 \mathrm{~m}$, fragment of wooden cover of pit burial no. 38. Est cal age $=3500 \pm 125 \mathrm{BP}$.

TB-249. Pevrebi

$3200 \pm 40$

Wood, depth $1.7 \mathrm{~m}$, fragment of wooden cover of pit burial no. 42. Est cal age $=3400 \pm 125 \mathrm{BP}$.

TB-250. Pevrebi

$2880 \pm 40$

Wood, depth $1.7 \mathrm{~m}$, fragment of wooden cover of pit burial no. 49. Est cal age $=3010 \pm 80 \mathrm{BP}$.

TB-251. Pevrebi

$2890 \pm 40$

Wood, depth $1.5 \mathrm{~m}$, fragment of wooden cover of pit burial no. 52. Est cal age $=3020 \pm 80 \mathrm{BP}$.

TB-252. Pevrebi

$2910 \pm 40$

Wood, depth $1 \mathrm{~m}$, fragment of wooden cover of pit burial no. 53. Est cal age $=3050 \pm 50 \mathrm{BP}$

TB-253. Pevrebi

$2950 \pm 40$

Wood, depth $1.5 \mathrm{~m}$, part of wooden cover of pit burial no. 57. Est cal age $=3100 \pm 80 \mathrm{BP}$.

TB-254. Pevrebi

$3180 \pm 45$

Wood, depth $1.5 \mathrm{~m}$, part of wooden cover of pit burial no. 59. Est cal age $=3400 \pm 125 \mathrm{BP}$. 
TB-256. Pevrebi

Wood, depth $1.5 \mathrm{~m}$, part of wooden cover of pit burial no. 68. Est cal age $=3300 \pm 125 \mathrm{BP}$.

TB-293. Pevrebi

Wood, depth $0.3 \mathrm{~m}$, part of wooden cover of pit burial no. 75. Est cal age $=2600 \pm 102 \mathrm{BP}$.

TB-294. Pevrebi

Wood, depth $1 \mathrm{~m}$, part of wooden cover of pit burial no. 76. Est cal age $=3200 \pm 125 \mathrm{BP}$.

TB-296. Pevrebi

Wood, depth $0.4 \mathrm{~m}$, part of wooden cover of pit burial no. 84 . Est cal age $=3500 \pm 125 \mathrm{BP}$.

TB-297. Pevrebi $3160 \pm 60$

Wood, depth $2 \mathrm{~m}$, part of wooden cover of pit burial no. 86 . Est cal age $=3400 \pm 125 \mathrm{BP}$.

TB-298. Pevrebi

Wood, base of pit, part of wooden cover of pit burial no. 86. Est cal age $=3300 \pm 125 \mathrm{BP}$.

TB-308. Udabno

Wood, depth $2 \mathrm{~m}$, part of wooden two-wheeled cart, burial mound no. 1. Est cal age $=3200 \pm 125 \mathrm{BP}$.

TB-328. Zeiani

Wood, depth $4 \mathrm{~m}$, part of wooden cover of burial mound no. 1. Est cal age $=5540 \pm 74 \mathrm{BP}$.

TB-329. Zeiani

$4740 \pm 75$

Wood, depth $3.5 \mathrm{~m}$, burial mound no. 1 . Est cal age $=5310 \pm 92 \mathrm{BP}$.

\section{Nakalakevi series}

Nakalakevi (Tsikhégoji) was one of centers of Colchian kingdom (W Georgia); later, in 4th to 8th centuries, Nakalakevi (Archaeopolis) became capital of Egrisian kingdom. This series includes samples from sites: 1) Nakalakevi, coll from king's bath, Nakalakevi village, Tskhakaya dist, Georgian SSR; 2) Shkhepi; Shkhepi castle is not far from Tskhakaya dist center (castle existed from 4th to beginning of 19th century); 3) Nojikhevi, $15 \mathrm{~km}$ from Nakalakevi village. Samples coll during excavations in palace and bath, Nojikhevi village, Gegechkori dist, Georgian SSR. Samples subm by P Zakaraya, State Mus Georgia.

TB-261. Nakalakevi

$1770 \pm 35$ 33BP.

Coal, depth 1 to $1.5 \mathrm{~m}$, Sq AD-25, Lot IX, bath. Est cal age $=1710 \pm$ 
TB-280. Nakalakevi

Coal, depth $1 \mathrm{~m}$, from under brick wall. Est cal age $=1570 \pm 41 \mathrm{BP}$.

TB-424. Nakalakevi

$1500 \pm 45$

Coal, depth $3.2 \mathrm{~m}$, from foundation of $\mathrm{E}$ tower of fortress. Est cal age $=1430 \pm 41 \mathrm{BP}$.

TB-425. Nakalakevi

$1860 \pm 45$ 33BP.

Coal, depth $2.8 \mathrm{~m}$, from base of fence ruins. Est cal age $=1810 \pm$

TB-426. Nakalakevi

$1890 \pm 40$ 33BP.

Coal, depth $3.5 \mathrm{~m}$, from vicinity of ancient gate. Est cal age $=1850 \pm$

TB-279. Shkhepi

$90 \pm 40$

Coal, depth $0.5 \mathrm{~m}$, on floor of 1 st story, main tower. Est cal age $=$ $160 \pm 42 \mathrm{BP}$.

TB-284. Shkhepi

$120 \pm 40$

Coal, depth $1 \mathrm{~m}$, remains of burned wall, main tower. Est cal age $=$ $180 \pm 42 \mathrm{BP}$.

TB-262. Nojokhevi

$1100 \pm 30$

Coal, depth 1 to $1.3 \mathrm{~m}$, bath. Est cal age $=1040 \pm 33 \mathrm{BP}$.

TB-263. Nojikhevi

$1100 \pm 30$

Coal, depth 1 to $1.5 \mathrm{~m}$, bath. Est cal age $=1040 \pm 33 \mathrm{BP}$.

TB-264. Nojikhevi

$980 \pm 30$

Coal, depth 0.5 to $1.5 \mathrm{~m}$, bath. Est cal age $=930 \pm 39 \mathrm{BP}$.

TB-265. Nojikhevi

$990 \pm 30$

Coal, depth 0.5 to $1.5 \mathrm{~m}$, bath. Est cal age $=940 \pm 39 \mathrm{BP}$.

TB-281. Nojikhevi

$200 \pm 40$

Coal, depth $0.6 \mathrm{~m}$, floor of palace chamber, Nojikhevi village. Est cal age $=250 \pm 42 \mathrm{BP}$

TB-282. Nojikhevi

$830 \pm 40$

Coal, depth 0.5 to $0.7 \mathrm{~m}$, floor of palace chamber, Nojikhevi village. Est cal age $=790 \pm 39 \mathrm{BP}$.

TB-283. Nojikhevi

$900 \pm 40$

Coal, depth $1 \mathrm{~m}$, floor of palace chamber, Nojikhevi village. Est cal age $=860 \pm 39 \mathrm{BP}$. 


\section{Treligoremi series}

TB-272. Treligoremi

Coal, depth $5 \mathrm{~m}$, chamber no. 1, ancient settlement no. 1, Treligoremi site, Dighomi residential dist, Tbilisi, Georgian SSR. Samples subm by R Abramishvili and Sh Iremashvili, Archaeol Inv Center, Hist, Archaeol \& Ethnog Inst, Acad Sci GSSR. Est cal age $=3020 \pm 80$ BP.

TB-273. Treligoremi 102BP.

Coal, depth $3.5 \mathrm{~m}$, from same loc as TB-272. Est cal age $=2800 \pm$

\section{TB-410. Treligoremi}

Coal, depth 0.75 to $0.8 \mathrm{~m}$, chamber no. 50 , ancient settlement no. 2, from same loc as TB-272. Est cal age $=2600 \pm 102 \mathrm{BP}$.

\section{Anaklia series}

\section{TB-274. Anaklia}

Wood, depth $8 \mathrm{~m}$, fragment of pillar, ancient settlement, Dikha-gudzuba II, Chitatskari site, Anaklia village, Zugdidi dist, Georgian SSR. Samples subm by D Muskhelishvili, Archaeol Inv Center, Acad Sci GSSR. Est cal age $=4200 \pm 109 \mathrm{BP}$.

\section{TB-275. Anaklia}

$3940 \pm 50$

Wood, depth 7.5m, floor board, Sec NO, Sq D6, from same loc as TB274. Est cal age $=4300 \pm 109 \mathrm{BP}$.

\section{TB-276. Anaklia}

$3760 \pm 50$

Cereals, depth $7.7 \mathrm{~m}$, remains of settlement, from same loc as TB-274. Est cal age $=4100 \pm 63 \mathrm{BP}$.

\section{TB-93. Dedoplis mindori}

$2220 \pm 40$

Wood, depth $2 \mathrm{~m}$, ceiling beams, altar hall of pagan temple, Dedoplis mindori site, Aradeti village, Kareli dist, Georgian SSR. Sample subm by I Gagoshidze, Hist, Archaeol \& Ehnog Inst, Acad Sci GSSR. Est cal age = $2210 \pm 49 \mathrm{BP}$.

\section{TB-223. Tetri-Tskaro}

$$
110 \pm 30
$$

Wood, remains of ruined wall of old bldg, Tetri-Tskaro dist, S Georgia. Sample subm by N Tsivtsivadze, Georgian Literature Chair, Tbilisi State Univ. Est cal age $=170 \pm 42 \mathrm{BP}$.

TB-227. Vani

$2310 \pm 40$

Coal, depth $0.45 \mathrm{~cm}$, Adeishvili gora, Mtisdziri, Vani dist, W Georgia. Sample subm by O Lortkipanidze, Hist, Archaeol \& Ethnog Inst, Acad Sci GSSR. Est cal age $=2320 \pm 39 \mathrm{BP}$. 
TB-246. Bambebi

$2640 \pm 45$

Coal, depth $4 \mathrm{~m}$, burned house, ancient settlement, Bambebi site, left bank of Mtkvari R, Uplistsikhé village, Gori dist, Georgian SSR. Sample subm by D A Khakhutaishvili, Hist, Archaeol \& Ethnog Inst, Acad Sci GSSR. Est cal age $=2700 \pm 102 \mathrm{BP}$.

TB-266. Tsalka

$620 \pm 40$

Charcoal, portable oven, daran (secret tunnel), Tsalka village, Tsalka dist, Georgian SSR. Sample subm by D Amiranashvili, Archaeol Inv Center, Acad Sci GSSR. Fst cal age $=610 \pm 28 \mathrm{BP}$.

\section{TB-285. Khorshi}

Coal, depth 0.5 to $0.6 \mathrm{~m}$, Khorshi village, Tskhakaya dist, Georgian SSR. Sample subm by G Grigolia, Archaeol Inv Center. Est cal age $=3970 \pm$ 63в

\section{TB-289. Zhinvali}

$3740 \pm 70$

Coal, depth 2.5 to $3 \mathrm{~m}$, altar, Site XVI, Zhinvali village, Dusheti dist, Georgian SSR. Sample subm by R Ramishvili, Archaeol Inv Center. Est cal age $=4080 \pm 63 \mathrm{BP}$.

\section{TB-326. Zhinvali}

Coal, depth $3.2 \mathrm{~m}$, charred logs, Site XXV, from same loc as TB-289. Est cal age $=6890 \pm 85 \mathrm{BP}$.

\section{TB-291. Kobuleti}

Wood, depth 3 to $4 \mathrm{~m}$, marshland, Kobuleti dist, Adjarian ASSR. Sample subm by I Melikadze, Mining Eng Inst, Acad Sci GSSR. Est cal age = $490 \pm 53 \mathrm{BP}$.

\section{TB-292. Jiéti}

$\mathbf{2 7 0 0} \pm \mathbf{5 0}$

Charcoal, depth $2.5 \mathrm{~m}, \mathrm{Sq}$ D-20, $\operatorname{Tr} 4$, remains of chamber 1, Jiétu site, Tsinsopeli village, Chiatura town, Georgian SSR. Sample subm by J Nadiradze, State Fine Arts Mus GSSR. Est cal age $=2800 \pm 102 \mathrm{BP}$.

\section{TB-299. Ureki}

$$
2600 \pm 200
$$

Wood, part of bronze axe handle (no. 953), burial pit 3, Ureki village, Makharadze dist, Georgian SSR. Sample subm by T Mikeladze, Archaeol Inv Center. Est cal age $=2700 \pm 102 \mathrm{BP}$.

\section{TB-301. Kachagani}

$6630 \pm 60$

Coal, depth $4.2 \mathrm{~m}$, at foot of wall 41, Khramis Didi Gora site, Kachagani village, Marneuli dist, Georgian SSR. Sample subm by D Gogelia, State Mus Georgia. Est cal age $=7250 \pm 73 \mathrm{BP}$.

\section{TB-322. Kachagani}

$6700 \pm 60$

Coal, depth $5.4 \mathrm{~m}, \mathrm{Sq}$ NV, ancient settlement, Kachagani village, Marneuli dist, Georgian SSR. Sample subm by T Kiguradze, State Mus Georgia. Est cal age $=7320 \pm 73 \mathrm{BP}$. 


\section{TB-315. Dzudzuni mgvimé}

Coal, 2nd layer, ancient cave settlement from Dzudzuni Mgvimé cave site, gorge of Kvirila R, Chiatura dist, Georgian SSR. Sample subm by L Zhorzhikashvili, Archaeol Inv Center. Est cal age $=6300 \pm 170 \mathrm{BP}$.

TB-316. Dzudzuni mgvimé

$4600 \pm 130$

Coal, 1st layer, ancient cave settlement, from same loc as TB-315. Est cal age $=5150 \pm 92 \mathrm{BP}$.

\section{TB-317. Martkopi}

$\mathbf{3 8 9 0} \pm \mathbf{5 0}$

Wood, depth $0.8 \mathrm{~m}$, part of wooden cover, burial mound 3, Martkopi village, Gardabani dist, Georgian SSR; subm by O Japaridze, Archaeol Chair, Tbilisi State Univ. Est cal age $=4300 \pm 109 \mathrm{BP}$.

TB-325. Martkopi

$4130 \pm 80$

Wood, depth $1.2 \mathrm{~m}$, part of wooden cover, burial mound 4 , from same loc as TB-317. Est cal age $=4600 \pm 108 \mathrm{BP}$.

TB-318. Chalagantené

$6580 \pm 60$

Coal, depth $1 \mathrm{~m}$, chamber of pottery kiln, Chalagantené settlement, Agdat dist, Azerbaijan SSR; subm by I Narimanov, Hist Inst, Acad Sci AzSSR. Est cal age $=7210 \pm 73 \mathrm{BP}$.

\section{TB-330. Namashevi tsikhé}

$1650 \pm 90$

Coal, depth $1 \mathrm{~m}$, Namashevi tsikhé site, Didi Gubi village, Tsulukidze dist, Georgian SSR; subm by V Japaridze, Archaeol Inv Center. Est cal age $=1590 \pm 41 \mathrm{BP}$.

\section{TB-336. Tsikhia Gora}

$2220 \pm 80$

Wood, depth 1.2m, chamber 15, Tsikhia Gora site, Kavtiskhevi village, Kaspi dist, Georgian SSR; subm by G Tskitishvili, Archaeol Inv Center. Est cal age $=2210 \pm 49 \mathrm{BP}$.

TB-337. Tsikhia Gora

$2180 \pm 60$

Coal, depth $1.2 \mathrm{~m}$, chamber 14 , from same loc as TB-336. Est cal age $=$ $2170 \pm 49 \mathrm{BP}$.

TB-411. Khrioki mitsebi

$1000 \pm 45$

Coal, depth $1.5 \mathrm{~m}$, remains of chamber, Khrioki mitsebi site, Vardisubani village, Dmanisi dist, Georgian SSR; subm by V Javaridze, Archaeol Inv Center. Est cal age $=950 \pm 39 \mathrm{BP}$.

TB-412. Khrioki mitsebi

$1530 \pm 45$

Coal, depth $1.6 \mathrm{~m}$, remnants of stone bldg, from same loc as TB-411.

Est cal age $=1460 \pm 41 \mathrm{BP}$. 
TB-413. Satsikhuris Gora

Coal, hearth 4, Satsikhuris Gora site, Tsagvli village, Khashuri dist, Georgian SSR; subm by A Ramishvili, Archaeol Inv Center. Est cal age = $3500 \pm 103 \mathrm{BP}$.

TB-414. Kvintsikhis Gora

$2520 \pm 50$

Coal, at level of chamber foundation, sample 1, Kvintsikhis Gora site, Kveda-Sakhano village, Zestafoni dist, Georgian SSR; subm by V Japaridze. Est cal age $=2600 \pm 102 \mathrm{BP}$.

TB-415. Kvintsikhis Gora

$2510 \pm 50$

Coal, chamber, sample 2, from same loc as TB-414. Est cal age = $2600 \pm 102 \mathrm{BP}$.

TB-416. Sachkheré

$4340 \pm 60$

Coal, depth $1.1 \mathrm{~m}$, adobe floor of upper bldg level, remains of structure, Argveti village, Sachkheré dist, Georgian SSR; subm by G Pkhakadze, Archaeol Inv Center. Est cal age $=4800 \pm 159 \mathrm{BP}$.

TB-417. Sachkheré

$4060 \pm 40$

Coal, depth 1.9 to $2 \mathrm{~m}$, log from pit, from same loc as TB-416. Est cal age $=4500 \pm 109 \mathrm{BP}$.

\section{TB-420. Satsikhuris Gora}

$\mathbf{3 3 8 0} \pm \mathbf{5 0}$

Coal, rectangular store room, upper level, Satsikhuris Gora site, Tsagvli village, Khashuri dist, Georgian SSR; subm by G Barabidze, Archaeol Inv Center. Est cal age $=3600 \pm 103 \mathrm{BP}$

TB-421. Gali

$2910 \pm 50$

Coal, depth $0.6 \mathrm{~m}$, remains of structure, 1st coal layer, Pichori village, Gali dist, Abkhazian ASSR; subm by M Baramidze, Archaeol Inv Center. Est cal age $=3050 \pm 80 \mathrm{BP}$.

\section{TB-422. Gedovani}

$15,700 \pm 120$

Bone, depth $1.5 \mathrm{~m}$, 3rd layer, Ortvala cave, Gedovani village, Terjola dist, Georgian SSR; subm by M Nioradze, Archaeol Inv Center.

\section{TB-423. Patardzeuli}

$340 \pm 40$

Wood, from bottom of cave in rocks between Patardzeuli and Khashmi villages, Sagarejo dist; Georgian SSR; subm by R Akhaladze, Tbilisi State Univ. Est cal age $=370 \pm 53 \mathrm{BP}$.

\section{TB-427. Ergeta}

$2520 \pm 50$

Wood, depth $0.8 \mathrm{~m}$, Naakargamusi tomb, Ergeta village, Zugdidi dist, Georgian SSR; subm by T Mikeladze. Est cal age $=2600 \pm 102 \mathrm{BP}$. 


\section{Gabrichkogo series, Czechoslovakia}

\section{TB-428. Gabrichkogo}

$8420 \pm 60$

Wood, oak log, depth $30 \mathrm{~m}$, in sand near bank of Danube R, Gabrichkogo village, Bratislava dist; subm by P Povinec, Comenius Univ, Bratislava.

TB-429. Gabrichkogo

Wood, oak log, depth $30 \mathrm{~m}$, from same loc as TB-428.

TB-430. Gabrichkogo

$8230 \pm 60$

Wood, oak log, depth $30 \mathrm{~m}$, from same loc as TB-428.

\section{HYDROLOGIC SAMPLES}

The Georgian SSR is one of the richest regions of the world in number and variety of mineral water springs. This can be explained by complexity of the geologic history and structure of Georgia's territory, creating favorable conditions for the formation and outcropping of mineral waters of most diverse nature. Georgia has over 1500 mineral water springs with total flow rate of $95,000,000 \mathrm{~L}$ per day. Of these springs $40 \%$ belong to bottling plants (Eristavi, 1966).

The Tbilisi ${ }^{14} \mathrm{C}$ Lab is studying ${ }^{14} \mathrm{C}$ and ${ }^{3} \mathrm{H}$ isotopes in carbonized mineral waters (Burchuladze et al, 1977, 1978) which are widely used for domestic and medicinal purposes. Much importance is given to origin, mixing of waters in different horizons, flow rate and extent of ${ }^{14} \mathrm{C}$ and ${ }^{3} \mathrm{H}$ concentration, which helps check pollution of mineral waters with sewage and rain waters. As a result of nuclear testing in the last 30 years, specific activity of ${ }^{3} \mathrm{H}$ and ${ }^{14} \mathrm{C}$ in the earth's atmosphere has sharply increased, making it possible to determine the age of mineral waters.

Two methods were used to extract carbonates from the mineral waters in order to determine ${ }^{14} \mathrm{C}$ directly at the spring:

1) trapping free carbonic acid by letting it pass through the sodium hydroxide solution;

2) separating carbonates dissolved in water through precipitation.

Analyses were made on samples of carbonized mineral waters from several Georgian districts-Borjomi, Pasanauri, Bolnisi, Tbilisi, Ujarma, Java, Tsagveri. Parameters, characterizing mineralization $(\mathrm{Mg} / \mathrm{l})$, chemical composition, and age of water samples (Jaliashvili et al, 1968), are given below.

\section{Georgian Mineral Water series}

TB-181. Borjomi

Gas, $\mathrm{CO}_{2}$, well 25, Kvibisi village.

TB-182. Borjomi

Water, $\mathrm{M}_{7.1} \mathrm{HCO}_{3} 84 / \mathrm{Na93}$, well 25, Kvibisi village. 
TB-183. Borjomi

Gas, $\mathrm{CO}_{2}$, well 41, Vashlovani village.

TB-184. Borjomi

Water, $\mathrm{M}_{6.1} \mathrm{HCO}_{3} 87 / \mathrm{Na} 86$, well 41, Vashlovani village.

TB-185. Borjomi

Gas, $\mathrm{CO}_{2}$, well 54, Likani village.

TB-186. Borjomi

Water, $\mathrm{M}_{5.7} \mathrm{HCO}_{3} 90 / \mathrm{Na} 83$, well 54, Likani village.

TB-187. Pasanauri

Gas, $\mathrm{CO}_{2}$, well 144, Pasanauri village.

TB-188. Pasanauri

$36,900 \pm 900$

Water, $\mathrm{M}_{8.9} \mathrm{Cl} 58 \mathrm{HCO}_{3} 42 / \mathrm{Na93}$, well 144, Pasanauri village.

TB-189. Bolnisi

$18,400 \pm 150$

Gas, $\mathrm{CO}_{2}$, well 1, Bolnisi village.

TB-190. Bolnisi

$20,900 \pm 200$ village.

Water, $\mathrm{M}_{8.5} \mathrm{HCO}_{3} 32 \mathrm{SO}_{4} 12 \mathrm{Cl} 12 / \mathrm{Ag} 23 \mathrm{Na} 6 \mathrm{Ca} 10 \mathrm{Fe}$, well 1, Bolnisi

TB-191. Tbilisi

$\geq 45,500$

Gas, $\mathrm{CO}_{2}$, well 8, Tbilisi.

TB-192. Tbilisi.

Water, $\mathrm{M}_{0.4} \mathrm{Cl} 49 \mathrm{SO}_{4} 18 \mathrm{HCO}_{3} 16 / \mathrm{Na} 87$, well 8, Tbilisi.

TB-193. Udjarma

Water, $\mathrm{M}_{8.6} \mathrm{Cl} 70 \mathrm{HCO}_{3} 28 / \mathrm{Na} 98 \mathrm{Ca}$ l, well 10, Udjarma village.

TB-196. Tsagveri

$33,800 \pm 370$

Water, $\mathrm{M}_{4.0} \mathrm{HCO}_{3} 86 / \mathrm{Na} 47 \mathrm{Ca} 22 \mathrm{Mg} 21$, well 1, Tsagveri village.

TB-194. Java, S Ossetian Autonomous region $\quad 31,300 \pm 350$ Water, $\mathrm{M}_{5.4} \mathrm{Cl} 58 \mathrm{HCO}_{3} 42 / \mathrm{Na} 74 \mathrm{Mg} 14 \mathrm{Ca} 12$, well 14, Java village.

TB-195. Java, S Ossetian Autonomous region

$18,240 \pm 260$

Water, $\mathrm{M}_{6.3} \mathrm{HCO}_{3} 52 \mathrm{Cl} 47 / \mathrm{Na} 88$ Cal 1, well 1, Mskhlebi village.

General Comment: samples TB-181, -183, -191, and -193 do not contain radioactive hydrogen and carbon isotopes. These samples are result of durable movement of underground water in water-bearing horizon, which led to decay of these isotopes.

Samples TB-187, -189, -194, -195, and -196 evidently show mixing of 
waters with younger waters from overlying horizons; absence of tritium signifies that waters of surface origin were not involved in mixing.

\section{SOIL SAMPLES}

Dating soil samples provides reliable information on humus regime and rates of carbon biologic metabolism. In this study we determined ${ }^{14} \mathrm{C}$ of subtropical podzols. Samples were collected from several levels (II to IV) of Kodori R profile, Ochamchira and Gulripshi districts, Abkhazian ASSR.

We prepared humic acids that are substances of relatively stable character from soil samples. Dates obtained confirm characteristic active circulation of substances formed during podzol formation and mobility of humus. Dating the organic portion of soil helps confirm the origin of podzol formation processes on these levels. Our data clearly illustrate the main soil formation processes. Samples subm by T M Subeliani, Soil Sci, Agrochem \& Land Reclamation Inst, GSSR Agric Ministry.

\section{Abkhazian ASSR series}

TB-201. Ganakhleba

$470 \pm 30$

Humic acid, 1st fraction, depth 0 to $10 \mathrm{~cm}$, Sec 11 , 2nd level, Ganakhleba village, Gulripshi dist.

TB-202. Ganakhleba

$\mathbf{3 3 0} \pm \mathbf{3 0}$

Humic acid, 2nd fraction, depth 0 to $10 \mathrm{~cm}$, Sec 11 , 2nd level.

TB-203. Ganakhleba

$5600 \pm 40$

Humic acid, 1st fraction, depth 50 to $60 \mathrm{~cm}$, Sec 11 , 2nd level.

TB-205. Ganakhleba

$3540 \pm 40$

Humic acid, 1st fraction, depth 20 to $30 \mathrm{~cm}$, Sec 11, 2nd level.

TB-206. Atara

$\delta^{14} \mathrm{C}=22.9 \pm 0.3 \%$

Humic acid, 1 st fraction, depth 0 to $15 \mathrm{~cm}$, Sec 2, 3rd level, Atara village, Ochamchira dist.

TB-208. Kindgi

$\delta^{14} \mathrm{C}=8.1 \pm 0.1 \%$

Humic acid, 1 st fraction, depth 0 to $15 \mathrm{~cm}$, Sec 4 , 4th level, Kindgi village, Ochamchira dist.

TB-209. Kindgi

$460 \pm 40$

Humic acid, 1st fraction, depth 20 to $30 \mathrm{~cm}$, Sec 4, 4th level.

TB-210. Atara

$\delta^{14} \mathrm{C}=10.5 \pm 0.15 \%$

Humic acid, 2nd fraction, depth 0 to $15 \mathrm{~cm}$, Sec 2, 3rd level.

TB-211. Atara

$330 \pm 40$

Humic acid, 1 st fraction, depth 20 to $30 \mathrm{~cm}$, Sec $2,3 \mathrm{rd}$ level. 
TB-212. Noushi

$$
\delta^{14} \mathrm{C}=16.2 \pm 0.2 \%
$$

Humic acid, 1 st fraction, depth 0 to $15 \mathrm{~cm}$, Sec 15 , 3rd level, Noushi village, Gulripshi dist.

TB-213. Noushi

Humic acid, 1st fraction, depth 20 to $30 \mathrm{~cm}$, Sec 15 , 3rd level.

TB-214. Kindgi

$\delta^{14} \mathrm{C}=7.0 \pm 0.1 \%$

Humic acid, 1 st fraction, depth 0 to $10 \mathrm{~cm}$, Sec 4 , 4 th level.

TB-215. Kindgi

$600 \pm 40$

Humic acid, 1st fraction, depth 40 to $50 \mathrm{~cm}$, Sec 4 , 4 th level.

\section{Mtskheta series, Georgian SSR}

TB-365. Mtskheta

$\delta^{14} \mathrm{C}=\mathbf{2 4 . 1} \pm \mathbf{0 . 3 \%}$

Humic acid, depth 0 to $20 \mathrm{~cm}$, meadow-brown soil, Mukhrani village, Mtskheta dist; subm by Ts Kobaidze, Agric Inst GSSR.

TB-366. Mtskheta

$250 \pm 120$

Humic acid, depth 40 to $60 \mathrm{~cm}$, from same loc as TB-365.

TB-367. Mtskheta

$\delta^{14} \mathbf{C}=23.7 \pm 0.3 \%$

Humic acid, depth 0 to $20 \mathrm{~cm}$, brown soil, Ksovrisi village, Mtskheta dist; subm by Ts Kobaidze.

TB-368. Mtskheta

$3400 \pm 740$

Humic acid, depth 40 to $60 \mathrm{~cm}$, from same loc as TB-367.

GEOLOGIC SAMPLES

TB-86. Poti, Georgian SSR

$7910 \pm 60$

Peat, depth $18 \mathrm{~m}$, on right bank of Rioni R, near Poti town; subm by Ch Janelidze, Geog Inst, Acad Sci GSSR.

TB-88. Kulevi, Georgian SSR

$4060 \pm 50$ lidze.

Peat, depth $5 \mathrm{~m}, 1.5 \mathrm{~km}$ S of Kuleti village, Khobi dist; subm by Ch Jane-

\section{TB-98. Pichora, Abkhazian ASSR}

$\mathbf{5 0 1 0}+\mathbf{5 0}$

Wood, depth $3 \mathrm{~m}$, from marine basal sediments, Pichora village, Gali dist; subm by Ch Janelidze.

\section{Supsa series, Georgian SSR}

TB-225. Supsa

$1940 \pm 50$

Decomposed wood, depth 19m, left bank of Rioni R, Supsa village, Lanchkhuti dist; subm by Ch Janelidze. 
TB-226. Supsa

Decomposed wood, depth $9 \mathrm{~m}$, from same loc as TB-225.

TB-228. Supsa

Decomposed wood, depth $19 \mathrm{~m}$, from same loc as TB-225.

TB-236. Mestia, Georgian SSR

$1030 \pm 40$

Decomposed peat, depth $1.75 \mathrm{~m}$, gorge of Nakra R, Mestia dist; subm by Ch Janelidze.

TB-237. Mestia

$2410 \pm 40$

Decomposed peat, depth $1.25 \mathrm{~m}$, basin of Nenskra R, Dombai-Lara marsh; subm by N Margalitadze, Bot Inst, Acad Sci GSSR.

TB-238. Borjomi, Georgian SSR

$\mathbf{5 8 1 0} \pm \mathbf{5 0}$

Decomposed peat, depth $10 \mathrm{~m}$, marsh, Dabadzevi plateau, Borjomi dist; subm by $\mathrm{N}$ Margalitadze.

TB-257. Sevan, Armenian SSR

$3350 \pm 45$

Peat, depth 5.1 to $5.2 \mathrm{~m}$, coll on shore of Sevan Lake, Bolshoy Sevan village, subm by M Tumanian, State Univ, Yerevan.

\section{Gagra series, Abkhazian ASSR}

TB-348. Gagra

$2130 \pm 45$

Peat, depth $0.90 \mathrm{~m}$, coastal area, Alakhadze village, Gagra dist; subm by Ch Janelidze.

\section{TB-350. Gagra}

$1930 \pm 45$

Peat, depth $1 \mathrm{~m}$, from same loc as TB-348.

TB-351. Gagra

$2300 \pm 45$

Peat, depth 1.25, from same loc as TB-348.

TB-349. Kazbegi, Georgian SSR

$4420 \pm 50$

Wood, depth $7 \mathrm{~m}$, coll on left bank of Tergi R, Ketrisi village, Kazbegi dist; subm by Ch Janelidze.

\section{Sukhumi series, Abkhazian ASSR}

Since 1979 research has been carried out in the coastal area of Sukhumi and adjoining shelf to estimate modern engineering geologic conditions and to prepare long-term predictions for change of these conditions during a period determined by economic tasks or amortization time $(50$ to $100 \mathrm{yr}$ ) of man-made structures.

Mollusk shells were collected from boring wells uncovering Holocene sediments in order to reconstruct paleogeog conditions of sediment accumulation of homogeneous lithologic layers.

All samples are mollusk shells, unless otherwise indicated. Samples 
subm by V G Jeiranashvili and R A Jokhadze, Sukhumi Group, Hydrogeol Party of 7th dist, Gidrospetsgeologia Prod Corp, USSR Geol Ministry.

TB-341. Sukhumi

$5180 \pm 60$

Sample 1, well 42, depth 16 to $17.5 \mathrm{~m}$.

TB-342. Sukhumi

$5590 \pm 60$

Sample 2, well 42, depth 33 to $34 \mathrm{~m}$.

TB-343. Sukhumi

$5380 \pm 60$

Sample 3, well 42 , depth 48.6 to $50.2 \mathrm{~m}$.

TB-344. Sukhumi

$5690 \pm 60$

Sample 4, well 42, depth 57 to $58 \mathrm{~m}$.

TB-345. Sukhumi

$5720 \pm 60$

Sample 3, well 42, depth 62 to $63 \mathrm{~m}$.

TB-346. Sukhumi

$9310 \pm 80$

Peat, well 721 , depth 26.2 to $26.7 \mathrm{~m}$.

TB-347. Pitsunda

$6210 \pm 60$

1 st sea level, depth $1.2 \mathrm{~m}$, Pitsunda resort, Gagra dist.

TB-352. Sukhumi

$6430 \pm 60$

Peat, Sample 1, well 61 , depth $8 \mathrm{~m}$, 1 st sea level.

TB-353. Sukhumi

$4040 \pm 50$

Sample 1, well 717, depth 3.1 to 3.8 .

TB-354. Sukhumi

$6060 \pm 60$

Sample 2, well 717, depth 6 to $6.9 \mathrm{~m}$.

TB-355. Sukhumi

$6050 \pm 60$

Sample 3, well 717, depth 6.9 to $8.1 \mathrm{~m}$.

TB-356. Sukhumi

$7960 \pm 70$

Sample 4, well 717 , depth 9.2 to $11.2 \mathrm{~m}$.

TB-357. Sukhumi

$4370 \pm 60$

Sample 1, well 716, depth 1.4 to 1.7 .

TB-358. Sukhumi

$6060 \pm 60$

Sample 2, well 716, depth 3 to $4 \mathrm{~m}$.

TB-359. Sukhumi

$6480 \pm 60$

Sample 3, well 716, depth 5 to $6 \mathrm{~m}$. 
TB-360. Sukhumi

$6540 \pm 60$

Sample 4, well 716, depth 7.1 to $8.2 \mathrm{~m}$.

TB-361. Sukhumi

$3340 \pm 50$

Sample 1, well 723, depth 4.5 to $6 \mathrm{~m}$.

TB-362. Sukhumi

$5540 \pm 60$

Sample 2, well 723, depth 10.5 to $11 \mathrm{~m}$.

TB-363. Sukhumi

$7630 \pm 80$

Sample 3, well 723, depth 13 to $13.7 \mathrm{~m}$.

TB-364. Sukhumi

$8690 \pm 80$

Sample 4 , well 723 , depth 18 to $18.4 \mathrm{~m}$.

TB-369. Sukhumi

$6520 \pm 70$

Well 50 , 1st sea level, depth 18 to $19 \mathrm{~m}$.

TB-370. Sukhumi

$10,900 \pm 100$

Well 724 , 1st sea level, depth 32.6 to $33.1 \mathrm{~m}$.

TB-371. Sukhumi

$4670 \pm 60$

Peat, sample 1, well 100, 1 st sea level, depth 8.3 to $8.5 \mathrm{~m}$.

TB-372. Sukhumi

$6590 \pm 70$

Peat, sample 2, well 100 , 1 st sea level, depth 11.1 to $11.3 \mathrm{~m}$.

TB-373. Sukhumi

$7500 \pm 70$

Well 41 , depth 14 to $15 \mathrm{~m}$.

TB-374. Sukhumi

$7860 \pm 70$

Well 48 , depth 38 to $39 \mathrm{~m}$.

TB-377. Sukhumi

$7140 \pm 70$

Sample 1, well 49 , depth 16 to $17 \mathrm{~m}$.

TB-375. Sukhumi

$7310 \pm 70$

Sample 2, well 49, depth 22 to $23 \mathrm{~m}$.

TB-376. Sukhumi

$6690 \pm 70$

Well 50, depth 20 to $21 \mathrm{~m}$.

TB-378. Sukhumi

$6920 \pm 70$

Well 63 , depth 24 to $25 \mathrm{~m}$.

TB-379. Sukhumi

$7210 \pm 70$

Sample 1, well 93 , depth 15 to $16 \mathrm{~m}$. 
TB-380. Sukhumi

$10,180 \pm 90$

Sample 2, well 93, depth 25 to $26 \mathrm{~m}$.

TB-381. Sukhumi

$13,500 \pm 130$

Well 97 , depth 13 to $14 \mathrm{~m}$.

TB-382. Sukhumi

$\mathbf{3 3 6 0} \pm \mathbf{5 0}$

Well 702 , depth 0.7 to $2.2 \mathrm{~m}$.

TB-383. Sukhumi

$7840 \pm 70$

Well 709 , depth 7 to $8.6 \mathrm{~m}$.

TB-384. Sukhumi

$2510 \pm 50$

Well 718 , depth 3.9 to $5.2 \mathrm{~m}$.

TB-385. Sukhumi

$6540 \pm 60$

Sample 1, well 722, depth 3.2 to $4.3 \mathrm{~m}$.

TB-386. Sukhumi

$7040 \pm 70$

Sample 2, well 722, depth 5.4 to 6.1 .

TB-387. Sukhumi

$7500 \pm 70$

Sample 3, well 722, depth 10.3 to $11.3 \mathrm{~m}$.

TB-388. Sukhumi

$3850 \pm 50$

Sample 1, well 724, depth 5 to $7.2 \mathrm{~m}$.

TB-389. Sukhumi

$5720 \pm 60$

Sample 2, well 724, depth 10 to $11.5 \mathrm{~m}$.

TB-390. Sukhumi

$5760 \pm 60$

Well 725 , depth 11.3 to $11.8 \mathrm{~m}$.

\section{REFERENCES}

Burchuladze, A A, Gedevanishvili, L D, Pagava, S V and Togonidze, G I, 1977, Radiocarbon variations in the atmosphere for period 1950-1975 obtained from analyses of wine spirits, in Internatl conf on low-radioactivity measurements and applications, The High Tatras, Oct 6-19, 1975, Proc: Bratislava, p 261-264.

Burchuladze, A A, Pagava, S V, Jurina, V, Povinec, P and Usacev, S, 1982, Short-term variations of radiocarbon during the last century, in Internatl conf on low radiocarbon activities '80, 2nd, The High Tatras 1980, Low-level counting, Proc: Bratislava, v 8, p 189194

Burchuladze, A A, Pagava, S V, Povinec, P, Togonidze, G I and Usacev, S, 1979, Short-term variations of cosmogenic radiocarbon with solar activity, in Internatl cosmic ray conf, 16th, Proc: Kyoto, Japan, v 3, MG sess I, p 200-205.

- 1980, Radiocarbon variations with the 11-year solar cycle during the last century: Nature, v 287, no. 5780, p 320-322.

Burchuladze, A A, Povinec, P, Usacev, S, Pagava, S V, Togonidze, G I, Eristavi, I V, Poloskova, A and Sivo, A, 1979, The high-purity counter gas filling and liquid scintillation solvents synthesis for high-precision radiocarbon measurements: Tbilisi Univ Press (in Russian).

Burchuladze, A A, Togonidze, G I, Eristavi, I V and Chalatashvili, D V, 1977, Investigation of mineral waters of Georgia by radiocarbon method, in Internatl conf on low-radioactivity 
measurements and applications, The High Tatras, Oct 6-19, 1975, Proc: Bratislava, $\mathrm{p}$ $383-386$.

- 1978, ${ }^{14} \mathrm{C}$ concentration determination in some mineral waters of Georgia, in USSR conf on astrophysical phenomena and radiocarbon, 6th, Oct 13-15, 1976, Proc: Tbilisi, p 401-406 (in Russian).

Burchuladze, A A, Togonidze, G I, Oganezov, P S and Pagava, S V, 1970, New system for the combustion of samples for radiocarbon dating, in Symposium on astrophysical phenomena and radiocarbon, Nov 25-27, 1969, Proc: Tbilisi, p 51-55 (in Russian).

Burchuladze, A A, Togonidze, G I, Pagava, S V and Eristavi, I V, 1974, On some method of synthesis of the scintillation solvent from natural patterns containing carbon, in USSR conf on astrophys phenomena and radiocarbon, 5 th, Tbilisi, Oct 4-6, 1973, Proc: Tbilisi, p 323-326 (in Russian).

Damon, P E, Long, A and Wallick, E J, 1972, Dendrologic calibration of the Carbon-1 4 time scale, in Rafter, TA and Grant-Taylor, T, eds, Internatl conf on radiocarbon dating, 8th, Wellington, New Zealand, p A29-A43.

Eristavi, D I, Mineral waters of Georgia: Tbilisi, Sabchota Sakartvelo Pub House (in Georgian).

Jaliashvili, V G, Meliava, F S and Chichua, T F, 1968, Hydrogeologic description of main medicinal mineral waters of the Georgian SSR, Proc: Health resort study and physiotherapeutics, Tbilisi, v 29, p 209-243 (in Russian). 